\title{
Effectiveness of coordination exercise in improving cognitive function in older adults: a prospective study
}

This article was published in the following Dove Press journal:

Clinical Interventions in Aging

28 September 2011

Number of times this article has been viewed

\author{
Timothy CY Kwok ${ }^{1,2}$ \\ KC Lam ${ }^{2}$ \\ PS Wong ${ }^{2}$ \\ WW Chau ${ }^{2}$ \\ Kenneth SL Yuen ${ }^{2,3}$ \\ KT Ting ${ }^{2}$ \\ Elite WK Chung ${ }^{2}$ \\ Jessie $\mathrm{CY} \mathrm{Li}^{2}$ \\ Florence $\mathrm{KY} \mathrm{Ho} \mathrm{Ho}^{2,4}$ \\ 'Department of Medicine and \\ Therapeutics, The Chinese University \\ of Hong Kong, ${ }^{2}$ Jockey Club Centre \\ for Positive Ageing, ${ }^{3}$ Laboratory of \\ Neuropsychology, Department of \\ Psychology, The University of Hong \\ Kong, ${ }^{4}$ Jockey Club CADENZA Hub, \\ Hong Kong SAR
}

Correspondence: Timothy CY Kwok Department of Medicine and

Therapeutics, Prince of Wales Hospital,

The Chinese University of Hong Kong,

Shatin, New Territories, Hong Kong SAR

Tel +85226323128

Fax +852 26373852

Email tkwok@cuhk.edu.hk
Background: Studies on the effect of a low intensity coordination exercise on the elderly with limited mobility are sparse. This prospective study attempted to compare the effectiveness of a customized coordination exercise and a strength exercise in improving the cognitive functioning and physical mobility on the elderly.

Methods: Participants from two centers for the elderly were allocated to practice either an 8 -week coordination training (CT) program or an 8-week towel exercise (TE) program. The Chinese Mini-Mental State Examination and Chinese Dementia Rating Scale (CDRS) were used to measure cognitive functioning of participants, and Timed Up-and-Go test for physical mobility. These assessments were administered before and after the program.

Results: Paired $t$-tests showed that the CDRS scores of the CT group improved significantly from 114.8 at pre-test to 119.3 after training $(P=0.045)$. The CDRS scores of the TE group also improved from 114.9 at pre-test to 116.9 after training.

Conclusion: Findings from this prospective study demonstrated that low-intensity level mindbody exercise could be beneficial to the cognitive functioning of older adults.

Keywords: coordination exercise, cognitive function, elderly, Chinese

\section{Introduction}

The benefits of physical exercise on cognitive function in the elderly have been demonstrated in many studies. Several large-scale longitudinal studies showed that older people who have a high level of physical activity, have a significantly lower risk of developing Alzheimer's disease and cognitive impairment. ${ }^{1-7}$ The results of a metaanalysis of 18 studies investigating the effectiveness of aerobic exercise concluded that fitness training could enhance the cognitive functioning of the elderly. ${ }^{8}$ This study also showed that a short duration, moderate-level training program could create an optimal effect on cognitive functions in the elderly. Another meta-analysis investigating the change of duration and intensity of physical activity conducted by van Gelder et al found that elderly people who participated in physical exercise for an average of 30 minutes per day or more could postpone their cognitive decline. ${ }^{9}$

However, studies on the benefits of physical training have focused closely on aerobic exercise such as walking, and strength exercise, such as weight lifting., ${ }^{4,6,710-12}$ These aerobic and strength exercises require the participants to be highly mobile. The elderly with low mobility, or who are hospitalized, might have difficulty enjoying the full benefit of the exercise because of their limited locomotive ability. Therefore exercise with reduced locomotion requirement, could provide the benefits of aerobic exercise to the elderly with restricted mobility. Recently, there has been growing 
research interest in the therapeutic effects of mind-body exercise. ${ }^{13,14}$ Tai Chi Chuan, commonly known as Tai Chi, is a typical example of mind-body exercise; it is characterized by slow motion and emphasizes the conscious control of body movements, ie, it requires less locomotive mobility and is deemed appropriate for most elderly people. ${ }^{15}$ Research has shown that the cognitive functions of the elderly could be well preserved with the aid of such mind-body exercise, in a way similar to typical physical exercise. ${ }^{3}$

Exercises with lower requirements of locomotive ability, such as coordination training (CT) and towel exercise (TE), are needed for the elderly with poor mobility. Both CT and TE require low locomotive ability, and thus are suitable for most elderly. The literature review showed that CT and TE may also be beneficial for the cognitive functioning of the elderly. The purpose of this study was to compare the effectiveness of CT and TE on the cognitive functioning and physical mobility of the elderly, with the aim of developing an exercise with a low mobility requirement, to benefit the cognitive functioning of the elderly. We hypothesized that the elderly in the CT group would show significant improvement in the cognitive measures compared with the elderly in the TE group.

\section{Method}

\section{Subjects}

Forty elderly (three male, 37 female) with normal cognition were recruited from two elderly centers of the Hong Kong Lutheran Social Service, aged 66-90 $($ mean $=79.0$, $\mathrm{SD}=5.8$ ).

Targeted participants were asked to take the Chinese version of Mini-Mental State Examination (CMMSE) as one of the screening criteria, and those who scored $\geq 18$ were eligible for this study. ${ }^{16}$ Other than that, there was no other inclusion or exclusion criterion in recruitment.

The ethics approval of this study was obtained from the Survey and Behavioural Research Ethics Committee of the Chinese University of Hong Kong. Participants confirmed their agreement to participate in this study by signing informed consent before the exercise began.

\section{Coordination training (CT)}

A physiotherapist from the Jockey Club Centre for Positive Ageing (JCCPA, see http://www.jccpa.org.hk) developed an 8-week exercise program, called Coordination Training (CT), which is a simplified version of Tai Chi. It focuses on the coordination and conscious control of the body. It was easy for the elderly to learn, and required a relatively low level of mobility to practice. The eleven movements included coordination of fingers, hands, eyes, and legs. A brief description of the eleven movements is set out in Table 1. The elements of Movement 4 are tabulated in Table 2 and graphically represented in Figure 1 as an example. Movement 4 helped to train participants' coordination of upper limbs, and was intended to imitate the movements of Tai Chi.

\section{Towel exercise (TE)}

The training protocol of TE was developed by the Leisure and Cultural Services Department, the Government of the Hong Kong Special Administrative Region in 2005. ${ }^{17}$ Similar to Movement 4 in CT, TE was a type of stretching exercise mainly to train upper limb and bilateral arm movements, but utilize a towel as a tool. It was strongly promoted by the government because it was easy for elderly people with various locomotive abilities to master. ${ }^{18} \mathrm{TE}$ benefited the elderly by improving circulation and helping to control weight, and aimed to reduce the chance of falling. ${ }^{19}$

Table I Coordination training: I I movements

\begin{tabular}{|c|c|c|}
\hline Movement & Brief description & $\begin{array}{l}\text { Coordination } \\
\text { to train }\end{array}$ \\
\hline 1 & $\begin{array}{l}\text { Looking forward when } \\
\text { head moves }\end{array}$ & Eye \\
\hline 2 & $\begin{array}{l}\text { Varying eye movement } \\
\text { when head moves }\end{array}$ & Eye \\
\hline 3 & $\begin{array}{l}\text { Pointing nose with fingers } \\
\text { Easy level: pointing with rest } \\
\text { Medium level: pointing } \\
\text { without rest } \\
\text { Difficult level: pointing with } \\
\text { eyes closed }\end{array}$ & Finger \\
\hline 4 & $\begin{array}{l}\text { Touching ears with fingers } \\
\text { Easy level: touching with rest } \\
\text { Medium level: touching } \\
\text { without rest } \\
\text { Difficult level: touching with } \\
\text { eyes closed }\end{array}$ & Finger \\
\hline 5 & $\begin{array}{l}\text { Palm switching facing } \\
\text { upwards and } \\
\text { downwards }\end{array}$ & Hand \\
\hline 6 & $\begin{array}{l}\text { Fingers pointing to designated } \\
\text { parts of body according } \\
\text { to coach instruction }\end{array}$ & Finger \\
\hline 7 & $\begin{array}{l}\text { Fingers pointing to each } \\
\text { other according } \\
\text { to coach instruction } \\
\text { Difficult level: pointing } \\
\text { with eyes closed }\end{array}$ & Finger \\
\hline 8 & Straightening fingers & Finger \\
\hline 9 & $\begin{array}{l}\text { Forming hand gestures according } \\
\text { to coach instruction }\end{array}$ & Finger \\
\hline 10 & $\begin{array}{l}\text { Forming body gestures according } \\
\text { to coach instruction }\end{array}$ & Hand and leg \\
\hline II & Heel and toe touching ground & Leg \\
\hline
\end{tabular}


Table 2 Coordination training: Movement 4

\begin{tabular}{|c|c|c|}
\hline Easy & Medium & Difficult \\
\hline I. Straighten two arms & I. Straighten two arms & I. Close the eyes \\
\hline 2. Point both index fingers to the front & 2. Point both index fingers to the front & 2. Straighten two arms \\
\hline $\begin{array}{l}\text { 3. Touch the left or right ear with } \\
\text { corresponding index finger, while the } \\
\text { other hand remains straight }\end{array}$ & $\begin{array}{l}\text { 3. Touch the left or right ear with } \\
\text { corresponding index finger, while the } \\
\text { other hand remains straight }\end{array}$ & $\begin{array}{l}\text { 3. Point both index fingers to the front } \\
\text { 4. Touch the left or right ear with } \\
\text { corresponding index finger, while } \\
\text { the other hand remains straight }\end{array}$ \\
\hline 4. Switch & 4. Switch & 5. Switch \\
\hline $\begin{array}{l}\text { 5. Practice ten cycles (left and right) } \\
\text { with I second rest between each cycle }\end{array}$ & $\begin{array}{l}\text { 5. Practice ten cycles (left and right) } \\
\text { without rest between each cycle }\end{array}$ & $\begin{array}{l}\text { 6. Practice ten cycles (left and right) } \\
\text { without rest between each cycle }\end{array}$ \\
\hline
\end{tabular}

\section{Procedure}

For the sake of convenience of participation and better monitoring of participants' progress, those in one center were allocated to practice $\mathrm{CT}$, and those in another center were allocated to practice TE. TE was chosen to compare with CT because these exercies were similar in a number of ways. Both exercises required subjects to follow instructions, and to coordinate upper limb and bilateral arm movements. Yet TE did not require such fine motor coordination as $\mathrm{CT}$.

CT and TE were conducted for 8 consecutive weeks, with one 40-minute session per week. Both groups had a 10-minute warm-up period at the beginning and a 10-minute cool-down period at the end of the session to prevent injury. The remaining 20 minutes would be taken up with the actual CT or TE exercise. Both exercise groups were conducted by qualified instructors trained by the physiotherapist, mentioned above.

For CT, there were three levels of difficulty: easy, medium, and difficult (see Table 2). The level of difficulty was increased mainly by reducing the rest time (demanding higher

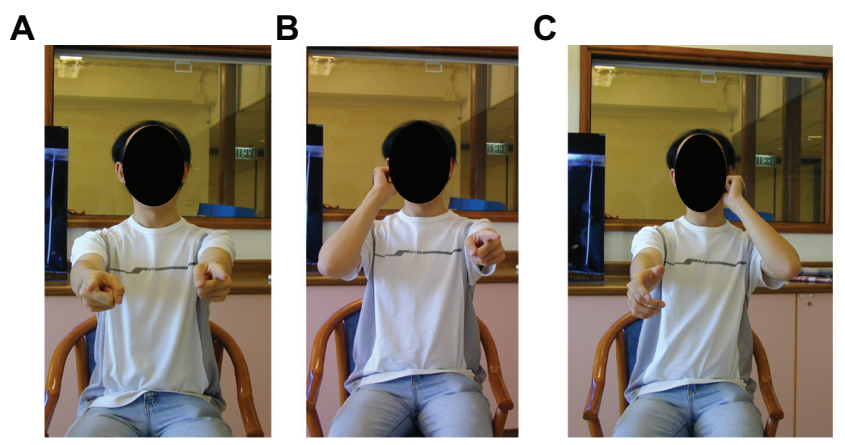

Figure I Graphical illustrations of Movement 4 of the coordination training. (A) Straighten two arms and point both index fingers to the front. (B) Touch the left or right ear with corresponding index finger, while the other hand remains straight. (C) Switch and practice ten cycles (left and right) with I second rest (easy) or without rest (medium and hard) between each cycle. concentration as well as physical strength of participants), and by closing the eyes when performing the actions (demanding higher psychomotor balance of participants). In this study, when the participants self-reported being able to handle the movement comfortably, which was confirmed by the trainer, they were required to practice the movement at an advanced difficulty level in order to avoid the ceiling effect. ${ }^{19}$

\section{Instruments}

Assessment tools including Chinese Mini-Mental State Examination (CMMSE), Chinese Dementia Rating Scale (CDRS), and Timed Up-and-Go test (TUG) were administered to participants in both groups before and after the training sessions by trained occupational therapists and clinical psychologists.

CMMSE and CDRS were used to assess participants' cognitive functioning. General cognitive status was assessed using the CMMSE, which was translated and validated by Chiu et al in the Hong Kong Chinese population. ${ }^{20}$ The full mark was 30 . The Cronbach's alpha was 0.86 . The test had excellent clinical utilities in screening general cognitive decline.

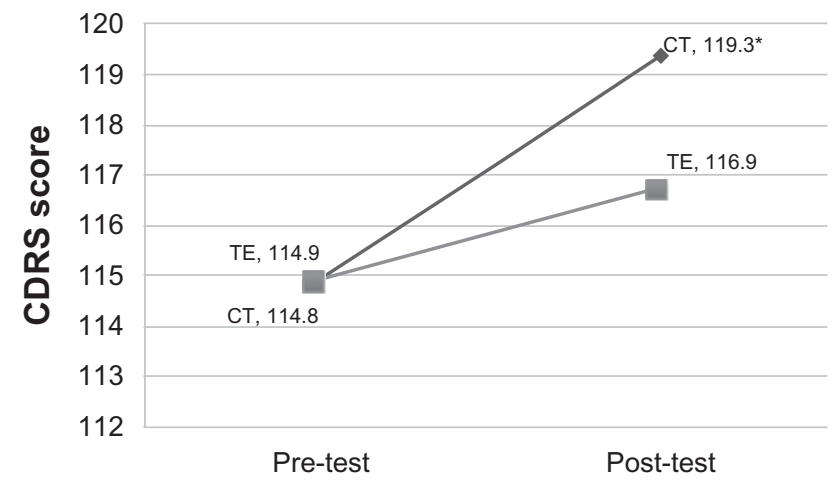

Figure 2 Changes in Chinese Dementia Rating Scale (CDRS) scores before and after the coordination training program (CT) or towel exercise program (TE) in respective groups.

Note: $* p<0.05$, paired samples $t$-test. 
CDRS measured the level of cognitive functioning of the participants. It examined five different aspects of cognitive ability, namely, attention, initiation-perseveration, construction, conceptualization, and memory. The maximum score of the unadjusted scale was 144 , with the Cronbach's alpha of 0.89 . Good psychometric properties were observed in both the original DRS and the Chinese version. ${ }^{21,22}$

TUG was a good instrument to measure the general physical mobility of participants, and thus was administrated in this study to measure the effects of relevant exercises. ${ }^{23}$ The longer time spent to finish TUG (slower), the poorer the performance of participants, and vice versa.

\section{Statistical analysis}

SPSS software v 15 (IBM Corp, Somers, NY) was used for data analyses. Independent sample $t$-tests were conducted to compare the pre-test scores (obtained in pre-test period) between CT and TE groups. Paired sample $t$-tests were performed to compare the post-test scores (obtained in the ninth week, after the 8-week exercise period) with the pre-test scores in each group. Analysis of covariance (ANCOVA) was used to compare the scores of CMMSE, CDRS, and TUG of the two groups after the training program, using participants' age and the pre-test scores as covariates. Statistical significant difference was set at $P<0.05$.

\section{Results}

Forty people (three males, 37 females) aged 66 to 90 years (mean $=79.0, \mathrm{SD}=5.8$ ) were recruited. The average ages of the elderly in the CT and TE groups were $77.7 \pm 6.0$ and $80.3 \pm 5.5$, respectively. Baseline characteristics are tabulated in Table 3. No significant difference was found in demographic features or cognitive and physical functioning test scores between the two groups.

Comparisons of participants' pre-test (baseline) and posttest cognitive functioning by CMMSE amd CDRS scores and physical mobility by TUG scores are shown in Table 4. Paired $t$-tests showed that the CDRS scores of the CT group had improved significantly from $114.8 \pm 15.5$ at pre-test to $119.3 \pm 18.0$ at post-test $($ CDRS $t(17)=-2.25, P=0.045)$. The CDRS scores of the TE group improved slightly from $114.9 \pm 14.8$ at pre-test to $116.9 \pm 12.5$ at post-test. No significant change was found in CMMSE $(t(18)=-0.931$, $P=0.368)$, and TUG $(t(17)=-0.334, P=0.747)$ in CT group, as well as CMMSE $(t(19)=0.665, P=0.516)$, CDRS $(t(19)=-0.891, P=0.384)$ and TUG $(t(19)=-1.908$, $P=0.086)$ in the TE group.
Table 3 Baseline characteristics of participants in CT and TE groups

\begin{tabular}{llll}
\hline Demographics & CT $(\mathbf{N}=\mathbf{2 0})$ & TE $(\mathbf{N}=20)$ & P value \\
\hline Gender $^{\mathrm{a}}$ (female \%) & 90.0 & 95.0 & 0.548 \\
Age $^{\mathrm{b}}$ & $77.7 \pm 6.0$ & $80.3 \pm 5.5$ & 0.153 \\
& $(66-86)$ & $(72-90)$ & \\
MMSE (pre-test) $^{\mathrm{b}}$ & $24.9 \pm 3.4$ & $25.6 \pm 2.5$ & 0.492 \\
& $(19-30)$ & $(20-30)$ & \\
Education level $^{\mathrm{a}}$ (N, \%) & & & \\
$\quad$ Illiterate & $6(30.0)$ & $8(40.0)$ & 0.459 \\
$\quad$ Informal education & $2(10.0)$ & $\mathrm{I}(5.0)$ & \\
$\quad \begin{array}{l}\text { Primary education } \\
\text { Secondary education }\end{array}$ & $7(35.0)$ & $7(35.0)$ & \\
$\quad$ University or above & $2(10.0)$ & $0(0.0)$ & \\
Missing & $3(15.0)$ & $0(0.0)$ & \\
\hline
\end{tabular}

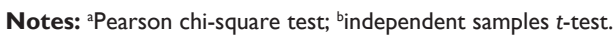
Abbreviations: CT, coordination training group; TE, towel exercise group; MMSE, Mini-Mental State Examination.

Different ANCOVA (between-subject factor: group [CT, TE] and covariates: age and the pre-test scores) models show the following findings. For CMMSE, the covariate age $\left(F(1,28)=0.17, P=0.690, \eta_{\mathrm{p}}^{2}=0.003\right)$ and the exercise groups $\left(F(1,28)=3.41, P=0.570, \eta_{\mathrm{p}}^{2}=0.139\right)$ were not significantly related to the CMMSE post-test scores. Only the covariate pre-test scores of CMMSE were significantly related to the post-test scores $\left(F(1,28)=16.32, P<0.001, \eta_{\mathrm{p}}{ }^{2}=0.428\right)$. For CDRS, exercise groups were not significantly related to the CDRS post-test scores $(F(1,28)=0.02, P=0.904$, $\left.\eta_{\mathrm{p}}{ }^{2}=0.001\right)$. Only the covariate age $(F(1,28)=9.14, P=0.005$, $\left.\eta_{\mathrm{p}}{ }^{2}=0.462\right)$ and the covariate pre-test scores of CDRS $\left(F(1,28)=59.12, P<0.001, \eta_{\mathrm{p}}{ }^{2}=0.738\right)$ were significantly related to the CDRS post-test scores. For TUG, the covariate age $\left(F(1,26)=0.01, P=0.940, \eta_{\mathrm{p}}{ }^{2}=<0.001\right)$ and the exercise groups $\left(F(1,26)=0.11, P=0.740, \eta_{\mathrm{p}}{ }^{2}=0.005\right)$ were not significantly related to the TUG post-test scores. Only the covariate pre-test scores of TUG were significantly related to the TUG post test scores $\left(F(1,26)=83.50, P<0.001, \eta_{\mathrm{p}}{ }^{2}=0.791\right)$.

\section{Discussion}

The above findings served to compare the effectiveness of the two exercise programs, coordination training (CT) and towel exercise (TE), in improving cognitive functioning and physical mobility in the elderly. The results showed that CT group participants had significant improvements in global cognition after the 8-week exercise program.

CT group gained significant improvement in CDRS scores after the exercise training, while the TE group participants did not. The lack of significant group difference in the changes in CDRS might be caused by the small sample size. Further investigation of the effectiveness of CT is 
Table 4 Means and standard deviations and the changes in CMMSE, CDRS, and TUG scores between pre-test (baseline) and post-test (eighth week) in CT and TE groups

\begin{tabular}{|c|c|c|c|c|c|c|c|c|c|}
\hline \multirow[t]{2}{*}{ Measures } & \multirow[t]{2}{*}{ Group } & \multirow[t]{2}{*}{$\mathbf{N}$} & \multicolumn{2}{|c|}{ Pre-test } & \multicolumn{2}{|c|}{ Post-test } & \multicolumn{2}{|c|}{ Change from pre-test } & \multirow[t]{2}{*}{$P$ value } \\
\hline & & & Mean & SD & Mean & SD & Mean & SD & \\
\hline \multirow[t]{2}{*}{ CMMSE } & CT & 19 & 24.9 & 3.4 & 25.8 & 3.6 & 0.9 & 2.9 & 0.368 \\
\hline & TE & 20 & 25.6 & 2.5 & 25.7 & 3.2 & -0.1 & 2.6 & 0.516 \\
\hline \multirow[t]{2}{*}{ CDRS } & $\mathrm{CT}$ & 18 & 114.8 & 15.5 & 119.3 & 18.0 & $4.6^{*}$ & 7.9 & $0.045^{*}$ \\
\hline & TE & 20 & 114.9 & 14.8 & 116.9 & 12.5 & 1.9 & 8.0 & 0.384 \\
\hline \multirow[t]{2}{*}{ TUG } & CT & 18 & 12.9 & 6.9 & 13.6 & 6.3 & 0.6 & 3.2 & 0.747 \\
\hline & TE & 20 & 14.7 & 7.0 & 15.7 & 8.1 & 0.9 & 1.8 & 0.086 \\
\hline
\end{tabular}

Note: $* P<0.05$

Abbreviations: CMMSE, Chinese Mini-Mental State Examination; CDRS, Chinese Dementia Rating Scale; TUG, Timed Up-and-Go test; CT, coordination training group; $\mathrm{TE}$, towel exercise group; SD, standard deviation.

recommended following this prospective study, through a large-scale clinical trial with appropriate numbers of samples in each group to detect the group differences.

For the physical mobility measure, TE tended to improve mobility while CT did not. This pattern was probably expected, because $\mathrm{CT}$ was designed to improve cognition, not mobility. There was also no significant difference between $\mathrm{CT}$ and TE after controlling for age. The insignificant difference in physical mobility measure might suggest that $\mathrm{CT}$, which required less in mobility, had a similar effect to TE, a common physical exercise, on the cognitive and physical functioning of the elderly population. The elderly with low mobility might benefit from physical exercise by practicing CT. Further investigation is needed to confirm this observation.

\section{Mind-body exercise to improve cognitive function}

Mind-body exercise can improve cognitive functions and other health indicators, although the role of physical exercise in modulating cognitive decline is complex. The improvements can be described through (1) psychosocial indicators and (2) physiological responses.

\section{Psychosocial indicators}

Practicing regular physical exercise was found to be associated with better cognitive test performance and decreased arousal. ${ }^{3,24}$ A moderate exercise program followed twice a week significantly slowed, by one-third, the progressive deterioration in ability to perform activities of daily living in people with Alzheimer's disease living in nursing homes. ${ }^{25}$ Mind-body exercises produce effects similar to those of regular cardiovascular exercises, suggesting an alternative model of exercise for the elderly, who are less able to exercise vigorously, to lower the risk of sport-related injuries and cardiac hazards.$^{15}$ Elderly people with the habit of regular physical exercise have been shown to be associated with socialization and environmental enrichment, which may also help attenuate the rate of cognitive decline. ${ }^{3}$ Tai Chi, a wellknown mind-body exercise, employs cognitive tools of both visualization and focused internal awareness to strengthen, relax, and integrate the body and mind. ${ }^{26}$ Tai Chi can also improve locomotion balance in seniors. ${ }^{27,28} \mathrm{~A}$ study evaluating a Tai Chi program called "Taiji (Tai Chi) Buddies Program" found that the program encouraged social participation and supported partner involvement, which may have a positive influence on exercise persistence and the health and wellbeing of the support partner. ${ }^{28} \mathrm{~A} 12$-week Tai Chi exercise program has been found adequate to reduce perceived stress and improve mood state, as well as increase perceived social support. ${ }^{29}$ The findings of this research showed that CT exercise, a simplified form of Tai Chi developed in this study specifically for the elderly with low activity, shares similar advantages, improving cognitive functions.

\section{Physiological responses to mind-body exercise}

Studies reviewing the physiological responses to mindbody exercise explain this phenomenon further. Mind-body exercise enhances cardiovascular function, muscle strength, body balance, and physical function; these improvements have a positive correlation with reduced stress, anxiety, and depression, resulting in an improved quality of life..$^{24,30,31}$ A study utilizing electroencephalogram (EEG) recorded an increased cordance value at left hemisphere (a sign of enhanced cerebral perfusion) in a patient with chronic epilepsy after practicing Dejian mind-body intervention (one of the components being mind-body exercise). ${ }^{32}$ The changes in brain activities reflected by EEG underlie the observed improvements in cognitive functions. ${ }^{32}$ In addition, practicing mind-body exercise, which exerts similar effects to aerobic exercise, helps to increase volume in 
both gray and white matters primarily located in prefrontal and temporal cortices - brain areas which are involved in age-related deterioration, as observed by MRI images. ${ }^{33}$ As demonstrated by animal models, exercise-induced upactive pathways are associated with enhancement of several neurotransmitter systems afferent to the hippocampus, including the norepinephrine, serotonin, acetylcholine, and $\gamma$-aminobutyric acid systems, which are important to hippocampal function. ${ }^{34}$ These changes in brain activities and functioning demonstrate that regular, moderate physical exercise has beneficial effects on brain health.

The findings of this study are consistent with previous reports that have shown that subjects practicing regular physical exercise are associated with better cognitive test performance, and there is a positive correlation between cardiovascular and mind-body exercise and cognitive function among the Chinese elderly. ${ }^{3,15}$ These exercises, however, might not be effective for the elderly suffering from moderate and severe dementia, who are likely to be immobile or even bed-bound. The "coordination training" exercise applied in this study, which requires a lesser level of physical movement, sheds light on improving cognitive functions for dementia patients who may find difficulty undertaking regular physical exercise because they are physically less active or less mobile. Additional, large-scale randomized control studies are recommended to elaborate on the efficacy of mind-body exercise on cognitive functioning.

\section{Limitations of this study}

The limitations of the study include the small sample size, and the absence of a control group (without any exercise). Participants in this study self-reported a habit of performing regular physical activities, and thus they are likely to be more health conscious with a lower cardiovascular burden. ${ }^{3}$

\section{Conclusion}

This prospective study attempted to provide evidence for the potential benefits of a customized coordination training exercise to improve the cognitive functioning of the elderly. The findings demonstrate that low physical level exercise similar to Tai Chi for example is beneficial for cognitive function and helps maintain the physical mobility of the elderly. The findings also give insight into developing further exercise regimes, which are more suitable for elderly people with a limited level of physical fitness or who are hospitalized. Additional research is encouraged to further confirm the effectiveness of the coordination training exercise.

\section{Disclosure}

The authors declare no conflicts of interest in relation to this paper.

\section{References}

1. Etgen T, Sander D, Huntgeburth U, Poppert H, Förstl H, Bickel H. Physical activity and incident cognitive impairment in elderly persons: the INVADE study. Arch Intern Med. 2010;170(2):186-193.

2. Geda YE, Roberts RO, Knopman DS, et al. Physical exercise, aging, and mild cognitive impairment: a population-based study. Arch Neurol. 2010;67(1):80-86.

3. Lam LC, Tam CW, Lui VW, et al. Modality of physical exercise and cognitive function in Hong Kong older Chinese community. Int J Geriatr Psychiatry. 2009;24(1):48-53.

4. Laurin D, Verreault R, Lindsay J, MacPherson K, Rockwood K. Physical activity and risk of cognitive impairment and dementia in elderly persons. Arch Neurol. 2001;58(3):498-504.

5. Podewils LJ, Guallar E, Kuller LH, et al. Physical activity, APOE genotype, and dementia risk: findings from the Cardiovascular Health Cognition Study. Am J Epidemiol. 2005;161(7):639-651.

6. Weuve J, Kang JH, Manson JE, Breteler MM, Ware JH, Grodstein F. Physical activity, including walking, and cognitive function in older women. JAMA. 2004;292(12):1454-1461.

7. Yaffe K, Barnes D, Nevitt M, Lui LY, Covinsky K. A prospective study of physical activity and cognitive decline in elderly women: women who walk. Arch Intern Med. 2001;161(14):1703-1708.

8. Colcombe S, Kramer AF. Fitness effects on the cognitive function of older adults: a meta-analytic study. Psychol Sci. 2003;14(2):125-130.

9. van Gelder BM, Tijhuis MA, Kalmijn S, Giampaoli S, Nissinen A, Kromhout D. Physical activity in relation to cognitive decline in elderly men: the FINE Study. Neurology. 2004;63(12):2316-2321.

10. Anderson-Hanley C, Nimon JP, Westen SC. Cognitive health benefits of strengthening exercise for community-dwelling older adults. $J$ Clin Exp Neuropsychol. 2010;32(9):996-1001.

11. Cassilhas RC, Viana VA, Grassmann V, et al. The impact of resistance exercise on the cognitive function of the elderly. Med Sci Sports Exerc. 2007;39(8):1401-1407.

12. Heyn P, Abreu BC, Ottenbacher KJ. The effects of exercise training on elderly persons with cognitive impairment and dementia: a metaanalysis. Arch Phys Med Rehabil. 2004;85(10):1694-1704.

13. Lan C, Lai JS, Chen SY, Wong MK. 12-month Tai Chi training in the elderly: its effect on health fitness. Med Sci Sports Exerc. 1998;30(3):345-351.

14. Lan C, Lai JS, Chen SY, Wong MK. Tai Chi Chuan to improve muscular strength and endurance in elderly individuals: a pilot study. Arch Phys Med Rehabil. 2000;81(5):604-607.

15. Chan AS, Ho YC, Cheung MC, Albert MS, Chiu HF, Lam LC. Association between mind-body and cardiovascular exercises and memory in older adults. JAm Geriatr Soc. 2005;53(10):1754-1760.

16. Kwok TCY, Chau WW, Yuen KSL, et al. Who would benefit from memory training? A pilot study examining the ceiling effect of concurrent cognitive stimulation. Clin Inter Aging. 2011.

17. Leisure and Cultural Services Department HKSAR. Healthy Exercise for All Campaign. Virtual Class for Healthy Exercise. Hong Kong: Leisure and Cultural Services Department HKSAR. Healthy Exercise for All Campaign; 2003 [updated May 23, 2007]. Available from: http:// www.lcsd.gov.hk/healthy/en/virtual.php. Accessed January 15, 2011.

18. wenweipo.com. Available from http://paper.wenweipo.com/2004/01/19/ OG0401190003.htm. Accessed January 18, 2011.

19. Taguchi N, Higaki Y, Inoue S, Kimura H, Tanaka K. Effects of a 12-month multicomponent exercise program on physical performance, daily physical activity, and quality of life in very elderly people with minor disabilities: an intervention study. $J$ Epidemiol. 2010;20(1):21-29. 
20. Chiu HFK, Lee HC, Chung WS, Kwong PK. Reliability of validity of the Cantonese version of Mini-Mental State Examination: a preliminary study. Journal of Hong Kong College of Psychiatry. 1994;4(2).

21. Chan AS, Choi A, Chiu H, Lam L. Clinical validity of the Chinese version of Mattis Dementia Rating Scale in differentiating dementia of Alzheimer's type in Hong Kong. J Int Neuropsychol Soc. 2003;9(1).

22. Chan AS, Poon MW, Choi A, Cheung MC. Dementia Rating Scale. Hong Kong: The Chinese University of Hong Kong; 2001.

23. Ng SS, Hui-Chan CW. The timed up and go test: its reliability and association with lower-limb impairments and locomotor capacities in people with chronic stroke. Arch Phys Med Rehabil. 2005;86(8):1641-1647.

24. La Forge R. Mind-body fitness: encouraging prospects for primary and secondary prevention. J Cardiovasc Nurs. 1997;11(3):53-65.

25. Rolland Y, Pillard F, Klapouszczak A, et al. Exercise program for nursing home residents with Alzheimer's disease: a 1-year randomized, controlled trial. J Am Geriatr Soc. 2007;55(2):158-165.

26. Wayne PM, Buring JE, Davis RB, et al. Tai Chi for osteopenic women: design and rationale of a pragmatic randomized controlled trial. BMC Musculoskelet Disord. 2010;11:40.

27. Ives JC, Sosnoff J. Beyond the mind-body exercise hype. Phys Sportsmed. 2000;28(3):67-81.
28. Klein PJ, Rivers L. Taiji for individuals with Parkinson disease and their support partners: a program evaluation. J Neurol Phys Ther. 2006;30(1):22-27.

29. Taylor-Piliae RE, Haskell WL, Waters CM, Froelicher ES. Change in perceived psychosocial status following a 12-week Tai Chi exercise programme. J Adv Nurs. 2006;54(3):313-329.

30. Wang C. Tai chi and rheumatic diseases. Rheum Dis Clin North Am. 2011;37(1):19-32.

31. Grodstein F. Cardiovascular risk factors and cognitive function. Alzheimers Dement. 2007;3(2 Suppl):S16-S22.

32. Chan AS, Sze SL, Cheung M-C, Lam JM, Shi D. Dejian mind-body intervention improves the functioning of a patient with chronic epilepsy: a case report. Cases Journal. 2009;2:9080.

33. Colcombe SJ, Erickson KI, Scalf PE, et al. Aerobic exercise training increases brain volume in aging humans. J Gerontol A Biol Sci Med Sci. 2006;61(11):1166-1170.

34. Ma Q. Beneficial effects of moderate voluntary physical exercise and its biological mechanisms on brain health. Neurosci Bull. 2008;24(4):265-270.
Clinical Interventions in Aging

\section{Publish your work in this journal}

Clinical Interventions in Aging is an international, peer-reviewed journal focusing on evidence-based reports on the value or lack thereof of treatments intended to prevent or delay the onset of maladaptive correlates of aging in human beings. This journal is indexed on PubMed Central, MedLine, the American Chemical Society's 'Chemical Abstracts

\section{Dovepress}

Service' (CAS), Scopus and the Elsevier Bibliographic databases. The manuscript management system is completely online and includes a very quick and fair peer-review system, which is all easy to use. Visit http://www.dovepress.com/testimonials.php to read real quotes from published authors. 